\title{
Una discusión sobre el rol de las Organizaciones Internacionales en la teoría de las Relaciones Internacionales
}

\author{
Juan Facundo Carcedo ${ }^{1}$ \\ Universidad Nacional del Centro de la Prov. de Bs. As. \\ Comisión de Investigaciones Científicas de la Prov. de Bs. As.
}

\section{Artículo Científico}

Material original autorizado para su primera publicación en el Journal de Ciencias Sociales, Revista Académica de la Facultad de Ciencias Sociales de la Universidad de Palermo.

\section{Recepción: 15-12-2018}

Aceptación: 17-03-2019

Resumen: El artículo tiene por objetivo dar cuenta de los diferentes enfoques que desde la disciplina de las Relaciones Internacionales permiten estudiar el fenómeno de las organizaciones internacionales. En este sentido, en primer lugar se realiza un abordaje conceptual y una caracterización de las organizaciones internacionales, así como una diferenciación del término Instituciones Internacionales. En segundo lugar, se señalan aquellos trabajos a los que se tuvo acceso que han estudiado el tema en los últimos años, evidenciándose que han existido esfuerzos desde diferentes puntos del planeta en indagar sobre este tema. En tercer lugar, se analiza el papel que juegan las organizaciones internacionales en la disciplina, específicamente desde los enfoques teóricos del realismo y neorrealismo, liberalismo, institucionalismo neoliberal e interdependencia compleja, constructivismo y otras contribuciones teóricas (entre ellas, la visión crítica, el funcionalismo, neo-funcionalismo, la teoría del agente-principal y la perspectiva feminista). Por último, se presentan reflexiones finales. Así, el artículo estudia un actor internacional considerado por los autores de carácter más estatocéntrico no tradicional en las Relaciones Internacionales, tal como son las organizaciones internacionales, que desde mediados del siglo XX han incrementado su presencia en el sistema internacional, vinculándose en la actualidad tanto

\footnotetext{
${ }^{1}$ Lic. en Relaciones Internacionales (FCH-UNICEN). Maestrando en Integración y Cooperación Internacional (CERIR/CEI-UNR). Estudiante de Doctorado en Relaciones Internacionales (UNR). Becario de la Comisión de Investigaciones Científicas de la Provincia de Buenos Aires (CICPBA). Miembro de la planta estable de investigadores del Centro de Estudios Interdisciplinarios en Problemáticas Internacionales y Locales (CEIPIL/CICPBA) de la Universidad Nacional del Centro de la Provincia de Buenos Aires (UNICEN). Correo electrónico: facundocarcedo@gmail.com
} 
con gobiernos nacionales como subnacionales y abarcando una amplia variedad de temas y problemáticas.

Palabras clave: organizaciones; internacionales; teoría; relaciones.

\section{A discussion on the role of the International Organizations in the International Relations theory}

Abstract: The article has as main objective to evidence the different approaches from the International Relations discipline that permit to study the international organizations. In this sense, first the article defines and characterizes the international organizations, as well as a differentiation from the term international institutions. Second, those researches published in the last years related to the issue of this paper are being exposed in the discussion section given account of the efforts made around the globe to inquire into this subject. Third, the article analyses the role played by the international organizations in the IR discipline, specifically from theories such as, realism and neo-realism, liberalism, neoliberal institutionalism and complex interdependence, constructivism, and others theoretical contributions (like the critical theory, the functionalism and neo-functionalism, the principal and agent, and the feminist theory). Finally yet importantly, final reflections are being presented. Thus, the paper studies an international actor considered by the most state-centric authors as non-traditional in the IR, such as the international organizations, which from mid- $20^{\text {th }}$ century have increased its presence in the international system, with currently relations with national and sub-national governments and reaching a huge variety of issues.

Keywords: organizations; international; theory; relations.

\section{Introducción}

El presente artículo tiene por objetivo dar cuenta de las diferentes perspectivas teóricas que posibilitan abordar el papel de las organizaciones internacionales en el sistema internacional.

Metodológicamente, se trata de un artículo de revisión teórico a partir de la utilización del método cualitativo y del análisis bibliográfico como técnica.

De esta forma, a los fines de este trabajo se realizó un estudio de las teorías de las Relaciones Internacionales, para seguidamente indagar sobre el papel de las organizaciones internacionales en la disciplina.

Teniendo en cuenta que las organizaciones internacionales pueden ser consideradas como actores no tradicionales en la disciplina, la cual ha tenido un marcado carácter 
estatocéntrico, en primer lugar, el trabajo realiza un repaso sobre las distintas definiciones de organizaciones internacionales, para, en segundo lugar, dar cuenta de aquellos trabajos a los que se tuvo acceso que estudiaron la temática. En tercer lugar, se realiza un abordaje de la forma en que cada teoría de las Relaciones Internacionales estudia las organizaciones internacionales, particularmente, la teoría realista y neo-realista, liberalismo, institucionalismo neoliberal y la interdependencia, el constructivismo y otras contribuciones teóricas, entre las que se encuentran la visión crítica, la teoría funcionalista y neo-funcionalista, la teoría agenteprincipal y el feminismo.

Por último, se presentan las reflexiones finales.

\section{Perspectiva teórica}

Resulta conveniente comenzar este apartado con algunas precisiones conceptuales, a fin de definir a qué nos referimos cuando hacemos mención a organizaciones internacionales y si es lo mismo hablar de instituciones internacionales.

En el presente trabajo se definen a las organizaciones internacionales como:

Asociaciones voluntarias de Estados establecidas por acuerdo internacional, dotadas de órganos permanentes, propios e independientes, encargados de gestionar unos intereses colectivos y capaces de expresar una voluntad jurídicamente distinta de la de sus miembros. (Diez de Velasco, 1997, p. 41)

Por su parte, Esther Barbé indica:

Una organización internacional es una asociación de estados establecida mediante un acuerdo internacional por tres o más estados, para la consecución de unos objetivos comunes y dotada de estructura institucional con órganos permanentes, propios e independientes de los estados miembros. (Barbé, 1995, p. 154)

A lo anterior debe agregarse que, para Barkin (2006), las organizaciones internacionales pueden ser definidas como "organizaciones intergubernamentales inclusivas" (Barkin, 2006, p. 1). Según este autor, son organizaciones que fueron creadas mediante acuerdo entre Estados antes que por individuos privados, y a las cuales todas las partes interesadas pueden adherir, diferenciándose así de las organizaciones intergubernamentales exclusivas $^{2}$ (Barkin, 2006).

Otra terminología ampliamente utilizada en la bibliografía en español e inglés consultada es "instituciones internacionales" como sinónimo para referirse a los actores bajo estudio.

${ }^{2}$ Las organizaciones intergubernamentales exclusivas están específicamente diseñadas para excluir algunos Estados (Barkin, 2006, p. 1). 
Así, de acuerdo con Thomas Risse (2002, p. 605, citado por Duffield, 2007, p. 1) "existen al menos tantas definiciones de instituciones internacionales como perspectivas teóricas". En concordancia con Duffield (2007) se puede señalar que de forma tradicional y frecuente se ha usado el término instituciones internacionales para hacer mención a organizaciones internacionales formales (tales como las instituciones financieras internacionales, es decir, el Fondo Monetario Internacional, en adelante FMI y el Banco Internacional de Reconstrucción y Fomento, en adelante BIRF). No obstante ello:

La equiparación de organizaciones con instituciones pudo haber tenido sentido en las décadas de 1950 y 1960 cuando las organizaciones internacionales eran el principal tema de investigación institucional de los académicos. A medida que otras formas institucionales internacionales, tales como regímenes, han proliferado en las tres décadas pasadas, sin embargo, tal construcción restrictiva del concepto ha devenido errónea, y como resultado, inapropiada. (Duffield, 2007, p. 3)

Por tal motivo, en el presente artículo, de carácter teórico, se opta por utilizar el concepto de organizaciones internacionales. En primer lugar, y como sinónimo, se utilizará la expresión organismos internacionales presente en la mayor parte de la bibliografía en español (no así en la inglesa). A fin de evitar confusiones teóricas, se prescinde de utilizar el término instituciones internacionales en vistas de lo señalado por Duffield (2007) con anterioridad.

A partir de las definiciones anteriores, es posible realizar una caracterización de las organizaciones internacionales, y señalar que poseen un carácter interestatal e intergubernamental, que se asientan en una base jurídica (reflejada en su convenio constitutivo), que se conforman de una estructura orgánica permanente y se apoyan en un esquema compuesto por una asamblea plenaria en la que participan todos los Estados miembros, así como una institución de composición restringida que asegura su gobierno y un secretariado encargado de su administración. Por último, se encuentran dotados de una autonomía jurídica independiente y distinta a la de sus Estados miembros, lo cual significa que están capacitados para elaborar o manifestar una voluntad autónoma en los ámbitos en los que gozan de competencias (Diez de Velasco, 1997).

Este último aspecto de las organizaciones internacionales fue uno de los más debatidos al interior de la disciplina, como se verá en los siguientes apartados.

\section{Discusión}

Entre los trabajos que han realizado abordajes sobre el papel de las organizaciones internacionales en la teoría de las Relaciones Internacionales pueden destacarse los de Barkin (2006), Pinto (2007), Zamudio González (2012), de Almeida Freitas Lacerda (2013) y Bayeh (2014). 
En este sentido, en primer lugar el libro de Samuel Barkin (2006) International Organization: Theories and Institutions realiza una conceptualización de las organizaciones internacionales y, seguidamente, aborda el estudio de las mismas desde las teorías realistas, institucionalistas, constructivistas y racionalistas, considerando a las organizaciones internacionales como agentes de cooperación internacional en el sistema internacional.

En segundo lugar, María do Ceu Pinto (2007) realiza un trabajo que se titula "Contributos das teorías das RI para o estudo das organizações internacionais e da integração regional" en el cual analiza el surgimiento del área de estudios de las organizaciones internacionales, así como la evolución y fases del estudio de estas. A continuación, la autora considera la teoría realista y neo-realista para luego continuar con aquellos enfoques que comenzaron en 1970, tales como la denominada interdependencia compleja (también llamada globalismo o transnacionalismo), el funcionalismo, neofuncionalismo y el constructivismo.

La autora concluye que "las teorías realistas y neo-realistas continúan siendo válidas para explicar ciertos aspectos del funcionalismo de las organizaciones internacionales, pero en muchos aspectos han sido ultrapasadas por el institucionalismo y el constructivismo" (Pinto, 2007, p. 99).

En tercer lugar, el libro Introducción al Estudio de las organizaciones internacionales gubernamentales: la pertinencia de una agenda de investigación interdisciplinaria de Laura Zamudio González (2012) se constituye en una importante referencia en la elaboración de este artículo.

Zamudio estudia las organizaciones internacionales gubernamentales en su rol como instrumentos (en el cual, al igual que el resto de los autores, aborda el realismo y el neorealismo, el funcionalismo y el neo-funcionalismo y la teoría de los regímenes), como actores burocráticos (donde analiza la visión burocrática weberiana de las organizaciones) y como actores desagregados (en el cual hace referencia a los procesos de toma de decisiones y la visión agente-principal).

En cuarto lugar, la publicación de Jan de Almeida Freitas Lacerda (2013) titulada "O papel das ideias e das Organizações Internacionais nas Teorias das Relações Internacionais" realiza una reflexión elaborada a partir de un análisis de la construcción teórica inserta en los grandes debates del campo de las Relaciones Internacionales, siendo notorio el diálogo entre las teorías neo-realistas, neo-institucionalista liberal y constructivista. Dentro de esa construcción, el autor se enfoca en la perspectiva de cada teoría mencionada acerca del papel de las ideas y de las organizaciones internacionales. 
Finalmente, otro de los estudios que desarrolla la temática de este trabajo es Endalcachew Bayeh (2014), quien en el artículo "Theories on the role of international organizations in maintaining peace and security" analiza las diferentes visiones sobre el rol de las organizaciones internacionales en el mantenimiento de la paz y la seguridad, recurriendo a la teoría constructivista, al institucionalismo liberal y al neo-realismo o realismo estructural.

De esta forma, el presente trabajo se suma a artículos que desde comienzos de la década del 2000 han estudiado el papel que ocupan las organizaciones internacionales en la teoría de las Relaciones Internacionales.

\section{Las organizaciones internacionales en la teoría de las Relaciones Internacionales}

El campo o sub-área de estudios de las organizaciones internacionales atraviesa los debates teóricos de las Relaciones Internacionales (De Almeida Freitas Lacerda, 2013, p. 85). Uno de los mayores problemas asociados con el estudio de las organizaciones internacionales en la teoría de las Relaciones Internacionales es la falta de un enfoque coherente hacia su comportamiento independiente en la política global (Andreev, 2007).

Así, a continuación se analiza la perspectiva realista (y sus reformulaciones), el institucionalismo neoliberal, el constructivismo y otras contribuciones tales como la visión crítica, el funcionalismo y neo-funcionalismo, la teoría del agente-principal y el feminismo.

\subsection{Realismo y Neorrealismo}

La corriente realista y sus posteriores reformulaciones consideran que el poder y la seguridad son los aspectos que realmente importan en el sistema internacional. A menudo recuerdan a las grandes guerras del pasado como ejemplo de los intentos fallidos de las organizaciones internacionales, como es el caso de la Liga de las Naciones.

Esta teoría de las Relaciones Internacionales analiza el rol de las organizaciones internacionales con escepticismo, ya que parten del presupuesto de que en el mundo contemporáneo los Estados son las organizaciones con mayor poder (Barkin, 2006). Este autor constata que:

Las organizaciones internacionales no poseen capacidades militares independientes y dependen de los Estados para hacer cumplir sus reglas. Al no tener capacidad para cobrar impuestos, dependen de los Estados para su financiamiento. Al no tener territorio, dependen de los Estados para albergarlas. De esta forma, las organizaciones internacionales pueden solamente tener éxito cuando son respaldadas por Estados poderosos. Entonces, para los realistas no tiene sentido centrar la atención en las organizaciones internacionales, porque las mismas reflejan 
la existencia del equilibrio de poder y los intereses de los Estados poderosos. Como resultado, tiene más sentido entender a las organizaciones internacionales como herramientas en la lucha de poder de los Estados, que como actores independientes. (Barkin, 2006, p. 8)

Teniendo en cuenta que en este trabajo se entienden las organizaciones internacionales como promotoras de la cooperación, para el realismo, la misma es:

Problemática pues los Estados desenvuelven sus actuaciones en un mundo que se considera de "suma cero": lo que uno gana el otro lo pierde. De esta forma el orden debe ser impuesto por el Estado dominante o, a veces, por Estados fuertes trabajando juntos. La ayuda externa sirve para que los Estados mantengan su poder y para su crecimiento, para lograr influencia política, prestigio, ventajas geoestratégicas, la intensificación del comercio, etc. (Ayllón, 2007, p. 35)

Con la reformulación neorrealista de los años ochenta, cuyo principal exponente es Kenneth Waltz, los Estados se constituyen en las principales categorías analíticas aunque, a diferencia del realismo tradicional, Waltz (2000) centra su explicación más en las características estructurales del sistema internacional y menos en las unidades que lo componen.

Waltz sostiene que "las instituciones internacionales son creadas y mantenidas por los Estados más poderosos para servir a sus intereses percibidos o no" (Waltz, 2000, p. 20) y que "los efectos que las instituciones internacionales pueden tener en las decisiones nacionales son efectos indirectos de las capacidades o intenciones del principal Estado 0 Estados que les dan nacimiento y las sustentan" (Waltz, 2000, p. 26).

Conforme indican Herz y Hoffman, los neorrealistas entienden que:

En la medida en que la cooperación, aunque presente en el sistema internacional, sea limitada por las condiciones de anarquía, el papel de las organizaciones internacionales como actores y, a veces, hasta como foros relevantes, es cuestionado. Las organizaciones internacionales gubernamentales no tienen poder ni autoridad para hacer que las decisiones sean cumplidas y los Estados optan por obedecer las reglas y normas creadas, de acuerdo con sus intereses nacionales. Las organizaciones son fundamentalmente instrumentos usados por los Estados más poderosos para alcanzar sus objetivos. Ellas sólo ejercen funciones importantes cuando expresan la distribución de poder en el sistema internacional. Apenas cuando los actores más poderosos acuerdan la utilización conjunta de las organizaciones internacionales gubernamentales para la realización de sus objetivos es esperado que ellas se tornen efectivas. (Herz y Hoffman, 2004, p. 50; citado en De Almeida Freitas Lacerda, 2013, p. 90)

Otro autor neorrealista, tal como es John Mearsheimer, sostiene que las organizaciones son el reflejo de la distribución del poder en el mundo y se encuentran basadas en cálculos egoístas de los grandes poderes, además de no tener efectos independientes en el comportamiento de los Estados (Mearsheimer, 1994/5, p. 7). 
A lo anterior, los neorrealistas agregan que: "Las instituciones internacionales son epifenómenos, lo cual significa que las Naciones Unidas, el FMI y la Unión Europea no tienen efectos independientes en los resultados internacionales. Los Estados usan a las organizaciones internacionales de forma instrumental, para su propia ganancia" (Meierhenrich, 2012, p. 26).

Asimismo, el neorrealismo posee una visión pesimista de la cooperación internacional, considerando que "los Estados se preocupan sumamente por su posición relativa (...) Un punto importante, que caracteriza el supuesto neorrealista, es su foco en las ganancias relativas" (Burchill, 2005, citado en Bayeh, 2014, p. 348). En este sentido, son los intereses estatales los que determinan la decisión de cooperar o competir (Bayeh, 2014).

A lo anterior se suma la conjetura neorrealista de que los Estados cooperan y adhieren a las organizaciones internacionales cuando les conviene (Sinclair y Byers, 2006). De esta forma, "las organizaciones internacionales sobreviven mientras permitan a los Estados seguir sus propios intereses y asistirlos en alcanzar ganancias relativas" (Bayeh, 2014, p. 348).

En suma, en el presente artículo se entiende que, si bien existen disparidades de poder en el sistema internacional, la perspectiva realista no permite realizar un estudio acabado de las organizaciones internacionales como actores con accionar autónomo, al menos legal o jurídico, de sus Estados miembros. La teoría realista recuerda tener en cuenta variables tales como qué Estado o Estados dieron origen a las organizaciones internacionales, y en qué contexto, de dónde proviene su financiamiento, entre otras cuestiones.

\subsection{Liberalismo, Institucionalismo neoliberal e interdependencia compleja}

Estas corrientes tienen sus orígenes en la teoría idealista, la cual "estima que la interdependencia económica lleva a la cooperación y la paz" (Jiménez González, 2003, p. 122). Las organizaciones internacionales permiten "instaurar mecanismos de cooperación en el mundo, los cuales podrán amortiguar los efectos de la anarquía internacional y reducir los conflictos entre naciones que culminen en guerras" (Jiménez González, 2003, p. 122).

En vista de lo anterior, este enfoque parte de la premisa de que el aumento de la interdependencia genera una mayor búsqueda de cooperación internacional. En consecuencia, las organizaciones internacionales surgen para solucionar los diferentes problemas de acción colectiva (Pinto, 2007).

De acuerdo con Pinto (2007), desde esta concepción:

Las organizaciones internacionales y los regímenes influyen significativamente en el comportamiento de los Estados y desempeñan funciones importantes, tales como: 
proporcionar información; reducen los costos de las transacciones; establecen reglas comunes para todos; refuerzan el cumplimiento de normas, establecen enlaces entre diferentes temas; mejoran la cooperación como resultado de la reducción de asimetrías o deficiencias de información; proveen oportunidades acrecidas de negociación y crean un ambiente de transparencia y de monitoreo necesario para el cumplimiento de las normas. (Pinto, 2007, p. 95)

Desde la percepción de los institucionalistas neoliberales, estas organizaciones tienen la función de constituirse en arenas o foros para la política de cooperación interestatal. Sin duda, asumen que los Estados no son los únicos actores que importan en la política mundial, consideran que las organizaciones internacionales, así como los acuerdos y regímenes internacionales, ayudan a cimentar la cooperación entre las naciones (Andreev, 2007).

No obstante lo anterior, el institucionalismo permanece estatocéntrico y considera que "las organizaciones internacionales en el mundo contemporáneo no son poderosos actores independientes" (Keohane y Nye, 1974, p. 39, citado por Andreev, 2007, p. 1). Aunque miran las organizaciones internacionales de forma diferente, tanto la perspectiva realista como la institucionalista se basan en el entendimiento de las organizaciones internacionales como actores intergubernamentales, es decir, actores que cooperan debido a los intereses compartidos por sus gobiernos nacionales, por consiguiente no siendo libres en su toma de decisiones (Andreev, 2007).

Desde la denominada interdependencia compleja (o enfoque transnacionalista), autores tales como Robert Keohane y Joseph Nye señalan que: "En la política de la interdependencia se encuentran involucrados intereses internos, transnacionales y gubernamentales. La política interna y externa comienza a eslabonarse estrechamente. La noción de interés nacional cada vez opone mayores dificultades para su uso eficaz" (Keohane y Nye, 1988, p. 22).

En este orden de ideas, los autores señalan que la interdependencia compleja posee tres características principales, a saber:

La sociedad se encuentra conectada por canales múltiples, los cuales se refieren a las relaciones interestatales, transgubernamentales y transnacionales $^{3}(\ldots)$. La agenda de las relaciones interestatales consiste en temas múltiples que no están colocados en una jerarquía clara o sólida. La diferencia entre temas internos y externos se vuelve difusa (...). La fuerza militar no es empleada por los gobiernos contra otros gobiernos de la región cuando predomina la interdependencia compleja. (Keohane y Nye, 1988, p. 41)

\footnotetext{
${ }^{3}$ Según Keohane y Nye $(1988$, p. 41) "las relaciones intergubernamentales incluyen aquellas que protagonizan las organizaciones intergubernamentales, creadas y mantenidas por Estados. Las relaciones tras-gubernamentales son interacciones directas entre subunidades de diferente Estados, mientras que las relaciones transnacionales son las interacciones a través de los Estados que no están controladas por los órganos centrales de política exterior de los gobiernos".
} 
Estos autores afirman que la presencia de canales múltiples lleva a predecir un diferente y significativo papel para las organizaciones internacionales en la política mundial.

De acuerdo con Ayllón, estos autores sostienen que "la cooperación internacional representa una forma menos costosa para que los Estados ejerzan su poder y, en algunos casos, su hegemonía" (Ayllón, 2007, p. 37). En este sentido, siendo optimista sobre la relevancia de las organizaciones internacionales, Keohane (1993) postula que las mismas son capaces de facilitar y constituirse en un marco para la cooperación.

Así, Keohane y Nye argumentan que las organizaciones internacionales contribuyen a establecer la agenda internacional, actuando como catalizadores para la formación de coaliciones y como escenario para iniciativas políticas y de vinculación entre los Estados débiles; en un contexto de creciente papel de las instituciones internacionales en la política internacional (Keohane y Nye, 1988).

Robert Keohane arguye en su obra "Instituciones Internacionales y Poder Estatal" que: "La capacidad de los Estados para comunicarse y cooperar depende de las instituciones hechas por el hombre, que varían históricamente y según los temas, en naturaleza y en fuerza" (Keohane, 1993, p. 15).

Se entiende por instituciones a "conjuntos de reglas (formales e informales) persistentes y conectadas, que prescriben papeles de conducta, restringen la actividad y configuran las expectativas" (Keohane, 1993, p. 17). Así, las instituciones internacionales asumen una de las siguientes tres formas:

- Organizaciones intergubernamentales formales 0 no gubernamentales internacionales, hacen referencia a organizaciones burocráticas con reglas explícitas y asignaciones específicas de reglas a individuos y grupos. Un ejemplo son las organizaciones intergubernamentales del sistema de Naciones Unidas.

- Regímenes internacionales, se refieren a instituciones con reglas explícitas, en las cuales han coincidido los gobiernos, que son pertinentes en conjuntos específicos de temas de relaciones internacionales. Por ejemplo, el régimen monetario internacional de Bretton Woods.

- Por último, convenciones, que son instituciones informales, con reglas y entendimientos implícitos. Un ejemplo es la reciprocidad (los líderes políticos esperan un tratamiento recíproco, tanto positivo como negativo). Antes de ser codificada en dos acuerdos internacionales, la inmunidad diplomática tradicional también se constituía en un ejemplo de convención (Keohane, 1993). 
A continuación, el autor sostiene que, sin encontrar excepciones, las organizaciones internacionales están inscriptas dentro de regímenes internacionales: gran parte de lo que realizan es controlar, manejar y modificar el funcionamiento de regímenes.

Además, Keohane (1993) sostiene que las organizaciones bajo estudio no poseen centralidad en la política mundial contemporánea, aunque son más importantes de lo que la teoría neorrealista ha reconocido.

En síntesis, desde el enfoque liberal las organizaciones internacionales desafían la idea de que el sistema internacional está basado en la necesidad de poder militar, ofreciendo posibilidades para la cooperación y la paz internacional. Si bien el Estado continúa detentando un lugar clave, este enfoque comienza a otorgar protagonismo a otros actores, entre los que se encuentran las organizaciones internacionales.

\subsection{Constructivismo}

El constructivismo, que irrumpió a finales de los años ochenta de la mano de Kratochwie (1989) y Onuf (1989), tuvo su influencia más significativa con el artículo de Alexander Wendt Anarchy is what States make of it (1992), y se constituye en una teoría de considerable valor para el presente estudio. La misma se concentra en el "rol de las ideas, normas, conocimiento y cultura, y debates en política, resaltando particularmente el rol de las ideas intersubjetivas y entendimientos de la vida social" (Finnemore and Sikkink, 2001, p. 392, citado en Duffield, 2007, p. 6).

Michael Barnett y Martha Finnemore (1999), exponentes de esta corriente, consideran a las organizaciones internacionales como actores autónomos en la política mundial ${ }^{4}$, y presentan un desafío a las teorías estatistas prevalecientes en las Relaciones Internacionales. A continuación, los autores se preguntan de dónde proviene la autonomía de las organizaciones internacionales, a lo que responden que la misma proviene de la legitimidad de la autoridad racional-legal y el control sobre la información y la expertise técnica.

Así, en primer lugar: "La autoridad racional-legal que las organizaciones internacionales encarnan le otorga un poder independiente de aquel de los Estados que las crearon y canalizan dicho poder en direcciones particulares" (Barnett y Finnemore, 1999, p. 699).

\footnotetext{
${ }^{4} \mathrm{Al}$ respecto, señalan que "las organizaciones internacionales son más que el reflejo de las preferencias del Estado y pueden ser autónomos y poderosos actores de la política global" (Barnett y Finnemore, 1999, p. 700).
} 
En este sentido, los autores explican que dicha autoridad deviene de la presencia de burócratas en las organizaciones internacionales, que desempeñan tareas administrativas e implementan normas establecidas racionalmente. Como consecuencia de ello, concluyen que las mismas son poderosas (Barnett y Finnemore, 1999).

En segundo lugar: "La autonomía de la burocracia deriva del conocimiento técnico especializado, entrenamiento y experiencia que no está inmediatamente disponible para otros actores" (Barnett y Finnemore, 1999, p. 708).

De esta forma, queda en evidencia que el análisis de las organizaciones internacionales desde la visión constructivista tiene como punto de partida la noción de burocracia (entendida en términos weberianos), en vistas de que la autoridad racional-legal y el control sobre la experticia son partes constitutivas y definen a cualquier burocracia (Barnett y Finnemore, 1999).

Así, en su obra Rules for the World, Barnett y Finnemore (2004) dan por tierra con la concepción realista de que las organizaciones internacionales representan los intereses y son controladas por los Estados más poderosos:

Entender las burocracias de las organizaciones internacionales abre una visión alternativa sobre las fuentes de su autonomía y qué pueden hacer con ella. Las burocracias no son meras servidoras de aquellos Estados que les delegan. Las burocracias son también autoridad por derecho propio, y esa autoridad les da autonomía vis-à-vis los Estados, individuos y otros actores internacionales (Barnett y Finnemore, 2004, p. 5).

Al igual que ocurre en las sociedades domésticas, la autoridad es conferida en diferentes grados y tipos, es decir, que el Estado tiene autoridad, pero también los académicos, profesionales y expertos, líderes de ONGs, organizaciones religiosas y personas de negocios (Barnett y Finnemore, 2004). En consecuencia, los autores señalan que: "En la esfera internacional, la autoridad también es conferida en diferentes grados y tipos, en otros actores diferentes de los Estados, entre ellos las organizaciones internacionales" (Barnett y Finnemore, 2004, p. 5).

A continuación, Barnett y Finnemore (1999) apuntan que las organizaciones internacionales cumplen más funciones que aquellas que les atribuye la corriente institucionalista neoliberal (aquellas relacionadas con superar fallas de mercado, dilemas de acción colectiva o problemas asociados con la interdependencia), sino que las organizaciones internacionales también cumplen la función de crear actores, especificar responsabilidades y autoridad entre ellas, al tiempo que definen el trabajo que esos actores deben realizar, brindando un significado y valor normativo. En este sentido, "Incluso cuando carecen de recursos materiales, las organizaciones internacionales ejercen poder al constituir y construir el mundo social" (Barnett y Finnemore, 1999, p. 700). 
Seguidamente sostienen que las organizaciones internacionales detentan autoridad y autonomía en el sistema internacional y que su poder (al igual que el de las burocracias en general) deviene "de que se presentan a sí mismas como impersonales, tecnocráticas y neutrales, como no ejerciendo poder, sino sirviendo a otros" (Barnett y Finnemore, 1999, p. 708).

A modo de ejemplo, resulta menester señalar la visión que el constructivismo aporta sobre el BIRF, cuando precisa que el mismo:

Es ampliamente reconocido por haber ejercido poder sobre las políticas de desarrollo muy superiores a su presupuesto (...). Después de décadas luego de su fundación, el Banco Mundial fue un imán para los mejores y más brillantes expertos del desarrollo. Su staff tuvo y continúa teniendo credenciales impresionantes de universidades prestigiosas y los elaborados modelos, informes y grupos de investigación que patrocinó a lo largo de los años tuvieron amplia influencia entre los expertos en el campo del desarrollo. Esta experticia, sumada a su reclamo de "neutralidad" y su estilo apolítico y tecnocrático de toma de decisiones, ha dado al Banco Mundial una voz autoritativa con la cual ha dictado exitosamente el contenido, dirección y alcance del desarrollo global a lo largo de los pasados cincuenta años. (Barnett y Finnemore, 1999, p. 709-710)

Luego, los autores se preguntan qué hacen las organizaciones internacionales con la autonomía y la autoridad. Para ello, examinan cómo aquellas clasifican el mundo, creando categorías de actores y acciones; fijando significado en el mundo social y articulando y difundiendo nuevas normas, principios y actores alrededor del globo (Barnett y Finnemore, 1999).

En otra de sus obras, Barnett y Finnemore (2004) han argumentado que las organizaciones internacionales poseen cuatro fuentes básicas de legitimidad, a saber, la autoridad racional-legal (explicada con anterioridad y que deviene de sus cartas), la legitimidad delegada (que deriva de los Estados), la legitimidad moral (que deviene de sus misiones importantes) y la legitimidad de la experiencia (basada en su trayectoria ampliamente aceptada en diferentes temas).

Al respecto, Daniel Béland y Mitchell Orenstein (2013) señalan que, en cuanto los enfoques realistas hacen hincapié en el aspecto racional-legal y la legitimidad delegada, los constructivistas enfatizan la legitimidad moral y de la experiencia, exponiendo que estas les otorgan a las organizaciones internacionales cierta autonomía con respecto a las agendas de los Estados más poderosos.

De esta forma, se puede considerar que este abordaje brinda las herramientas teóricas que se requieren en el presente trabajo para analizar el accionar de una organización internacional, considerando a la misma como un actor autónomo de los Estados que la crearon. 


\subsection{Otras contribuciones teóricas}

Desde una visión crítica de las Relaciones Internacionales encarnada por el canadiense Robert Cox, quien retoma los conceptos de Antonio Gramsci ${ }^{5}$, es posible afirmar que la hegemonía mundial se describe como una estructura social, económica y política. La misma se expresa en normas, instituciones y mecanismos universales que pueden establecer reglas generales de comportamiento (que apoyan el modo dominante de producción) para los estados y para las fuerzas de la sociedad civil que actúan de forma internacional (Cox, 1983).

En este orden de ideas, Cox considera que es más apropiado hablar de orden mundial en lugar de relaciones internacionales, debido a la necesidad de ir más allá de las relaciones interestatales y superar el estatocentrismo de la disciplina. En el orden mundial los Estados constituyen solo un componente más (Cox, 2001).

Además, destaca que,

Un mecanismo a través del cual se expresan las normas universales de la hegemonía mundial son las organizaciones internacionales. De hecho, las organizaciones internacionales funcionan como el proceso a través del cual se desarrollan las instituciones de la hegemonía y sus ideologías. Entre las características de la organización internacional que expresan su papel hegemónico se encuentran las siguientes: 1) encarnan normas que facilitan la expansión de órdenes hegemónicos mundiales; 2) son ellas mismas producto de órdenes hegemónicos mundiales; 3) legitiman ideológicamente las normas de un orden mundial; 4) cooptan a las elites de los países periféricos; y 5) absorben ideas contra-hegemónicas. (Cox, 1983, p. 62)

Asimismo, Cox indica que las instituciones internacionales son generalmente iniciadas por el Estado que establece la hegemonía. Por tal motivo, se puede afirmar que para la teoría crítica las organizaciones internacionales son meras herramientas usadas por los Estados económicamente más poderosos para imponer su control e influencia sobre Estados menos económicamente fuertes.

Por otra parte, menciona que las instituciones internacionales (y entre ellas las organizaciones internacionales) ejecutan un papel ideológico, ya que ayudan a definir lineamientos de políticas para los Estados y para legitimar ciertas prácticas e instituciones a nivel nacional (Cox, 1983).

Desde la teoría funcionalista ${ }^{6}$ de las organizaciones internacionales, iniciada por David Mitrany en A Working Peace System (1943), se consideraba que el ideal del gobierno

\footnotetext{
${ }^{5}$ Es importante destacar que para Gramsci (1971), el Estado continúa siendo la entidad básica de las relaciones internacionales y el lugar donde ocurren los conflictos sociales.

${ }^{6}$ Es preciso aclarar que la misma permite analizar el proceso de integración regional, al igual que sus desarrollos posteriores, como el neo-funcionalismo.
} 
mundial no sería alcanzado por medio de la disminución de las soberanías nacionales, sino por el desarrollo gradual de la cooperación internacional en áreas técnicas (Pinto, 2007), en la cual las organizaciones internacionales cumplirían un rol clave, ya que se encuentran "basadas en la función y no en el territorio, que permitirán la satisfacción de esas necesidades o problemas comunes" (Rodríguez Manzano, 1996, p. 296).

En consonancia con este desarrollo teórico, en 1947 fue creada la revista de referencia en el área, International Organization, que propició el desarrollo de esta área de investigación, con la explosión del fenómeno de las organizaciones internacionales con el fin de la Segunda Guerra Mundial.

Con posterioridad, en la década del sesenta, la teoría neo-funcionalista, desarrollada desde la Universidad de Berkeley por un grupo de especialistas guiados por Ernst Haas, propuso que los cambios a nivel tecnológico y científico producirían incentivos y presiones para la innovación institucional internacional, la cual llevaría al aprendizaje político de las organizaciones internacionales, las burocracias nacionales y de los líderes políticos (Malamud, 2011).

A diferencia del funcionalismo, el neo-funcionalismo incluye una paulatina transferencia de soberanía de los Estados hacia una entidad supranacional, tal como señala Barrea (1991) "el neo-funcionalismo destruye las soberanías políticas existentes para recompensar una nueva a nivel superior de la sociedad" (Barrea, 1991, p. 248; citado en Rodríguez Manzano, 1996, p. 301).

Otro aporte para el presente estudio es la teoría del agente-principal, que sostiene la existencia de un actor (el principal) que delega autoridad a un cuerpo específico (el agente), particularmente diseñado para realizar ciertas tareas. De esta forma la teoría argumenta que:

Cuando los Estados crean organizaciones internacionales les delegan autoridad en ciertas áreas y los enganchan en una relación agente-principal en la que ellos son los principales. El mayor problema analítico lo explica entonces el margen de maniobra de los agentes. (Reinalda y Verbeek, 2004, p.21, citado en Zamudio González, 2012, $\mathrm{n} / \mathrm{d})$

El agente recibe concesiones condicionadas de autoridad de un principal, para que este actúe en su nombre y le ayude a realizar funciones que le son importantes y costosas en términos unilaterales. Sin embargo, tal como recuerda Hawkins et al (2006, p. 7, citado en Zamudio González, 2012, n/d) "esta concesión está claramente limitada por la voluntad del principal y puede ser revocable".

Por último, desde un abordaje feminista de las organizaciones internacionales se analiza la falta de representatividad del género femenino en las mismas, así como critica la 
visión masculina de la realidad internacional que poseen $y$, por ende, los asuntos que incorporan en sus agendas (Hurtado Hurtado, 2014).

\section{Conclusión}

En el presente artículo se analizó el papel de las organizaciones internacionales en la teoría de las Relaciones Internacionales. Tal como fue posible evidenciar, existe una amplia discusión a nivel teórico sobre el rol de éstas en la disciplina.

Del análisis realizado se desprende la conveniencia de realizar en futuros trabajos de investigación la lectura de la realidad internacional y de las organizaciones internacionales a la luz de la corriente constructivista con aportes de la interdependencia compleja. Dicha corriente teórica considera a las organizaciones internacionales como actores jurídicamente independientes de aquellos Estados que le dieron origen, tomando distancia del enfoque estatocéntrico en el estudio de las mismas.

Por su parte, la interdependencia compleja otorga un rol preponderante a las organizaciones bajo estudio, en la búsqueda de la cooperación internacional, posibilitando la vinculación entre diferentes actores no tradicionales en la disciplina de las Relaciones Internacionales, tales como las empresas transnacionales y los gobiernos subnacionales.

Así, queda en evidencia cómo desde la disciplina se ha tratado de dar cuenta del creciente incremento en la participación internacional de las organizaciones internacionales destinadas a diversos fines.

\section{Referencias Bibliográficas}

Andreev, A. (2007). To what extent are international organizations (IOs) autonomous actors in world politics? Opticon1826, 2, 1-8.

Ayllón, B. (2007). La Cooperación Internacional para el Desarrollo: fundamento y justificaciones en la perspectiva de la Teoría de las Relaciones Internacionales. Carta Internacional, 2(2), 25-40.

Barbé, E. (1995). Relaciones Internacionales. Madrid: Tecnos.

Barkin, J. S. (2006). International Organization: Theories and Institutions. New York: Macmillan.

Barnett, M. y Finnemore, M. (1999). The Politics, Power, and Pathologies of International Organizations. International Organization, 53(4), 699-732.

Barnett, M. y Finnemore, M. (2004). Rules for the World: International Organizations in Global Politics. Ithaca: Cornell University Press.

Barrea, J. (1991). Théories des Relations Internationales. París: CIAO. 
Bayeh, E. (2014). Theories on the role of international organizations in maintaining peace and security. International Journal of Multidisciplinary Research and Development, 1(7), 347-350.

Béland, D. y Orenstein, M. (2013). International organizations as policy actors: An ideational approach. Global Social Policy. 13(2), 125-143.

Burchill, S. (2005). Liberalism. En S. Burchill et al (2005), Theories of International Relations (pp. 55-83). Nueva York: Macmillan.

Cox, R. (1983). Gramsci, hegemony and international relations: an essay in method. Journal of International Studies, 12(2), 162-175.

Cox, R. (2001). The Way Ahead: Towards a New Ontology of World Order. En R. Wyn Jones, Critical Theory and World Politics (pp. 45-60). Boulder: Lynne Rienner.

De Almeida Freitas Lacerda, J. (2013). O papel das ideias e das Organizações Internacionais nas Teorias das Relações Internacionais. Revista de Estudos Internacionais, 4(1), 83-99.

Diez de Velasco, M. (1997): Instituciones de Derecho Internacional Público. España: Tecnos. Duffield, J. (2007). What are International Institutions? International Studies Review, 9(1), 122.

Finnemore, M. y Sikkink, K. (2001). Taking Stock: The Constructivist Research Program in International Relations and Comparative Politics. Annual Review of Political Science, 4, 391-416.

Gramsci, A. (1971): Selections from the Prison Notebooks. New York: International Publishers.

Hawkins, D. et al (2006): Delegation and Agency in International Organizations (Political Economy of Institutions and Decisions). Cambridge: Cambridge University Press.

Herz, M. y Hoffmann, A. (2004): Organizações Internacionais: História e Práticas. Rio de Janeiro: Elsevier.

Hurtado Hurtado, J. (2014). Feminismo y organizaciones internacionales, ¿cambio o continuidad en las estructuras de poder? Revista Mito Cultural, 44 (n/d).

Jiménez González, C. (2003). Las teorías de la cooperación internacional dentro de las relaciones internacionales. Polis, 2(3), 115-147.

Keohane, R. (1993). Instituciones internacionales y poder estatal. Ensayos sobre teoría de las relaciones internacionales. Argentina: Grupo Editor Latinoamericano.

Keohane, R. y Nye, J. (1988). Poder e interdependencia. La política mundial en transición. Buenos Aires: Grupo Editor Latinoamericano.

Kratochwil, F. (1989). Rules, Norms and Decisions: On the Conditions of Practical and Legal Reasoning in International Relations and Domestic Affairs. Cambridge: Cambridge University Press. 
Malamud, A. (2011). Conceptos, teorías y debates sobre la integración regional. Norteamérica, 6(2), 219-249.

Mearsheimer, J. (1994/95). The False Promise of International Institutions. International Security, 19(3), 5-49.

Meierhenrich, J. (2012). International Organizations. Londres: London School of Economics and Political Science (LSE).

Mitrany, D. (1943). A working peace system. Chicago: Quadrangle Books.

Onuf, N. (1989). World of Our Making: Rules and Rule in Social Theory and International Relations (New International Relations). Londres: Routledge.

Pinto, M. (2007). Contributos das teorías das RI para o estudo das organizações internacionais e da integração regional. Relações Internacionais, 16(n/d), 85-101.

Reinalda, B. y Verbeek, B. (2004). Decision Making Within International Organizations. Londres: Routledge.

Risse, T. (2002). Constructivism and International Institutions: toward conversations across Paradigms. En I. Katznelson y H. Milner (Eds.), Political Science: The State of the Discipline (pp. 597-623). Nueva York: Norton.

Rodríguez Manzano, I. (1996). Funcionalismo, Neofuncionalismo y Relaciones Internacionales. Dos contribuciones doctrinales al estudio de las organizaciones internacionales. Dereito, 5(1), 289-303.

Sinclair A. y Byers M. (2006). When US Scholars Speak of "Sovereignty", What Do They Mean? TranState Working Papers, 44(n/d), 1-26.

Waltz, K. (2000). Structural Realism after the Cold War. International Security, 25(1), 5-41.

Wendt, A. (1992). Anarchy is what States make of it: the Social Construction of Power Politics. International Organization, 46(2), 391-425.

Zamudio González, L. (2012). Introducción al Estudio de las organizaciones internacionales gubernamentales: la pertinencia de una agenda de investigación interdisciplinaria. México: Centro de Investigación y Docencia Económica. 